UMD-PP-99-014

\title{
Limits on Pauli Principle Violation by Nucleons
}

\author{
E. Baron ${ }^{1}$, Rabindra N. Mohapatra ${ }^{2}$, and Vigdor L. Teplitz ${ }^{3}$ \\ (1) Department of Physics and Astronomy, University of Oklahoma, Norman, \\ OK-73019, USA. \\ (2) Department of Physics, University of Maryland, College Park, MD-20742, USA. \\ (3) Department of Physics, Southern Methodist University, Dallas, TX-75275, USA
}

\begin{abstract}
We consider nuclei produced in core collapse supernovae and subjected to a high neutron flux. We show that an accelerator mass spectrometry experiment that searched for traces of anomalous iron isotopes could set limits on the order of $10^{-20}-10^{-25}$ on (or perhaps discover) Pauli principle violation by neutrons. A similar search for anomalous Co isotopes could set limits in the range $10^{-13}-10^{-18}$ on Pauli principle violation by protons. We show that existing data on Oxygen can be used to set a limit of about $10^{-17}$ in one proposed model of such violation.
\end{abstract}


The Pauli exclusion principle, one of the the fundamental principles on which our basic understanding of the inner workings of systems ranging from atoms and nuclei to solids and liquids all the way to the stars and the universe rests, is clearly valid to a high degree of precision. Two simple examples in the domain of chemistry and physics are the patterns observed in the periodic table and the success of the shell model for nuclei. Until recently, however, there were hardly any quantitative tests of this fundamental principle. The best limit on possible deviation from the Pauli principle before 1987 was from an early experiment of Goldhaber and ScharffGoldhaber [i] in which beta rays from $\mathrm{C}^{14}$ were allowed to impinge on lead and a search was made for K-shell x-rays. This bound on possible violation of the Pauli principle (characterized by a parameter $\beta^{2}$ in this article and earlier [2]) by electrons was at the level of 3\%. In 1968, Fishbach, Kirsten and Schaffer [3] conducted a search for anomalous ${ }^{9} \mathrm{Be}$ and put an upper limit on the atmospheric density of such elements. Their result could be interpreted [2] to derive an upper limit of $\beta^{2} \leq 2 \times 10^{-3}$.

In 1987-90, several theoretical papers postulated ways to incorporate small deviations from Bose and Fermi statistics into quantum mechanics and field theories [4, 2, 5, 6]. At this time, it was noted [2] that atomic spectroscopy could improve this bound for electrons to the level of $10^{-7}$. Inspired by the theoretical interest in the subject, several dedicated experiments were planned around 1987-88 and have now considerably improved the limits for electrons [7, 8, 9]. These limits are now at the level of $10^{-26}-10^{-27}$. As far as other particles go, strong limits for protons (though much weaker than for electrons) have been extracted by Plaga [10] from considerations of energy generation in the Sun. We have listed these limits in the first four rows of Table 1. Note that to date no limit has been established for neutrons. There also exist limits on deviations from bose statistics for bosonic systems such 
as pions [2], photons [11] and spin zero atoms [12], which we do not address here.

Meanwhile, on the theoretical front it was shown that deviation from Fermi and Bose statistics can be embodied in a satisfactory manner [6] using the following "quonic" commutation relations [5] among the annihilation and creation operators $\left(a_{i}, a_{i}^{\dagger}\right)$ :

$$
a_{i} a_{j}^{\dagger}-q a_{j}^{\dagger} a_{i}=\delta_{i j}
$$

The Hilbert space of states for this model is positive definite, though it leads to a non-local field theory [6] and a number operator which is non-polynomial. Also we note that for the fermionic particles, $q=-1+\beta^{2}$.

In view of the fundamental role of the Pauli principle, it appears to us of considerable interest to expand the domain of our knowledge of the limits on Pauli violation (PV for short) to as many particles as possible.

The purpose of this note is to point out potential, high precision tests of the Pauli principle, for neutrons and protons, making use of the high neutron and proton fluxes in a type II supernova and also to derive a bound from already existing limits on anomalous oxygen isotope by Hemmick et al. [13]. At the risk of removing all suspense, we have included our results from this paper in Table 1. 
Table 1. Pauli Principle Tests

\begin{tabular}{llll}
\hline Particle & Reference & Method & Upper limit on $\beta^{2}$ \\
\hline Electron & Goldhaber and Goldhaber [1] & K-capture & 0.03 \\
Electron & Fishbach et al [3] & Anomalous ${ }^{9} B e$ & $2 \times 10^{-3}$ \\
Electron & Greenberg and Mohapatra [2] & Spectroscopy & $10^{-7}$ \\
Electron & Kelleher [0] & Spectroscopy & $10^{-7}$ \\
Electron & Ramberg and Snow [8] & Anomalous X-rays & $10^{-27}$ \\
Electrons & Pomansky et al. [9] & Mass spectrometry & $10^{-26}$ \\
Proton & Plaga [1] & Solar p-p cycle & $10^{-15}$ \\
Neutron & This paper & Supernova Oxygen \& & $10^{-17}$ \\
& & Mass spectrometry & (model-dependent) \\
Neutron & This paper & Supernova Iron \& & $10^{-25}$ \\
& & Mass spectrometry & is possible \\
Proton & This paper & Supernova Cobalt \& & $10^{-18}$ \\
& & Mass spectrometry & is possible \\
\hline
\end{tabular}

Our argument is as follows. About half of the terrestrial iron is produced during core collapse of massive stars to form Type II (or Type Ib/c) supernovae [14]. There, nickel is produced and there is also a significant flux of free neutrons. After the supernova bounce, the ensuing shock wave ejects the nickel which decays to cobalt which, in turn, decays to iron. In the supernova explosion there is approximately a fraction of a second during which the nickel is formed and exposed to a high flux of neutrons with thermal energies of about $0.5 \mathrm{MeV}$. The exact value of the neutron flux depends on the electron fraction of the material ejected $Y_{e}$, which in turn depends on the uncertain details of the supernova process. The other half of the terrestrial iron is produced in Type Ia supernovae by the same process, but the density is lower and the free neutron density is significantly lower since the supernova occurs as a thermonuclear explosion of a $\mathrm{C}+\mathrm{O}$ white dwarf and there is little time for weak interactions to occur. Thus we will concentrate on iron produced in core collapse 
supernovae. During the explosion, Pauli violation (PV) could permit the process

$$
n+{ }^{A-1} N i \rightarrow{ }^{A} N i^{*}+\gamma
$$

where ${ }^{A} N i^{*}$ contains an extra neutron in an already occupied shell model state. For example it could have 3 neutrons in the $1-s$ shell. Assuming that this (inner) configuration was not disturbed in the electron capture transitions from $N i^{*}$ to $F e^{*}$ (and did not inhibit those transitions), we would have today about the same relative abundance, up to a factor of two, of anomalous iron. Normal stable iron isotopes have $\mathrm{A}=54,56,58$. The unstable isotopes with $\mathrm{A}=55$ and 59 are the products of shortlived isotopes and also have very short halflives. Hence they are not expected to occur in terrestrial radioactivities. Thus experimental detection of these two, which is well within the capabilities of accelerator mass spectrometry, would be an indication of Pauli violation. The abundances of ${ }^{54} \mathrm{Fe}$ and ${ }^{58} \mathrm{Fe}$, from which ${ }^{55} \mathrm{Fe} e^{*}$ and ${ }^{59} \mathrm{Fe}^{*}$ would be made, effectively, are $5.9 \%$ and $0.33 \%$.

We turn now to the question of what abundance ratio, $F e^{*} / F e$, we can expect for a given amount of Pauli violation (PV). The probability that a neutron will be captured into a PV state is given by

$$
P_{V}=\beta^{2} \sigma n v t
$$

where $\beta^{2}$ measures the suppression of the cross section $\sigma$ that would obtain in the absence of the Pauli principle, $n$ is the neutron density, $v$ is the velocity of the neutrons, and $t$ is the time available.

There should be three contributions to the cross section for the process of $\mathrm{Eq}(2)$ : magnetic dipole radiation from neutron spin flip; magnetic dipole radiation from Ni spin flip; and electric dipole radiation from Ni. Blatt and Weisskopf 15 give a useful discussion of capture and disintegration cross sections which we follow. We keep only the first contribution (neutron spin flip) since the others are smaller, 
and we attempt to be conservative in estimating the rate. We use the zero range approximation (Eq (4.27) of [15]). We ignore possible scattering enhancement factors, i.e. $(1-a \gamma)^{2}$ where $a$ is the scattering length and $\gamma=(2 \mu B)^{1 / 2}$ with $\mu$ the n-Ni reduced mass, about the nucleon mass, and $B$ the anomalous binding energy, on the order of $20 \mathrm{MeV}$. The capture cross section is then

$$
\begin{aligned}
\sigma & =\pi\left(e^{2} / \hbar c\right)\left(\hbar c / M_{n} c^{2}\right)^{2}\left(B / M_{n} c^{2}\right)\left(2 B / E_{n}\right)^{1 / 2} \\
& =10^{-30} \mathrm{~cm}^{2}
\end{aligned}
$$

where $E_{n}=0.5 \mathrm{MeV}$ is the neutron energy. For the density of neutrons, we have attempted to be conservative in our estimate, and are guided by the results from assuming nuclear statistical equilibrium (NSE) (see Table 2 for some typical values in the region of the core that is ejected by the shock wave based on the model of Cooperstein, Kahana and Baron[16]).

In the supernovae the material is not ejected with NSE abundances, but rather undergoes an alpha rich freeze out that has been suggested to be in quasi-statistical equilibrium [17] (more alpha particles at a given $\left(\rho, \mathrm{T}, Y_{e}\right)$ than would be predicted by NSE). The neutron and proton densities drop precipitiously with temperature as the material expands. While the neutron flux is not determined by NSE, it is indicative. As the temperature drops during expansion, NSE is an increasingly poor assumption and so one has to both use a QSE code and know the expansion, T, and Ye history of the material, which is totally uncertain. Therefore the number $X_{n}$ used by us is simply a guess "guided" by the results in Table 2 which is based on assuming a reasonable equation of state for supernova material. We take $\rho \sim 10^{9} \mathrm{~g} \mathrm{~cm}^{-3}$, and a neutron mass fraction $X_{n} \sim 10^{-4}$, giving $n \sim 10^{29} \mathrm{~cm}^{-3}$. The thermal velocity of $0.5 \mathrm{MeV}$ neutrons is about $10^{9} \mathrm{~cm} \mathrm{~s}^{-1}$ and the $\mathrm{Ni}$ is exposed to neutrons for about a 0.1 second. This gives

$$
P_{V} \sim 10^{8} \beta^{2}
$$


That is, we have

$$
\beta^{2}<10^{-8} \mathrm{~EB}
$$

where EB stands for an experimental upper bound on the relative abundance of anomalous iron. The above estimate is for the dominant isotope of iron i.e. ${ }^{56} \mathrm{Fe}$. Since we are interested in ${ }^{59} F e^{*}$, we must multiply this probability by the factor $3 \times 10^{-3}$ to take into account the low abundance of ${ }^{58} \mathrm{Fe}$ (which presumably implies the corresponding low abundance of the parent $N i$ isotope which participated in the PV reaction). Based on experience with such bounds for other elements [13], we can expect that limits in the range $10^{-13}$ to $10^{-18}$ are readily achievable. Such an experiment would then set a PV limit for $\beta^{2}$ on the order of $3 \times 10^{-19}$ to $3 \times 10^{-24}$. If we use a similar argument for anomalous ${ }^{55} \mathrm{Fe}^{*}$, we would consider a parent $\mathrm{Fe}$ nucleus to be ${ }^{54} \mathrm{Fe}$ whose abundance is $5.9 \%$. Thus, looking for ${ }^{55} \mathrm{Fe} e^{*}$ would provide bound of $1.5 \times 10^{-20}$ to $1.5 \times 10^{-25}$.

Table 2. Illustrative Abundances for Supernova Conditions

\begin{tabular}{llllllll}
\hline$\rho\left(\mathrm{g} \mathrm{cm}^{-3}\right)$ & $\mathrm{T}(\mathrm{MeV})$ & $Y_{e}$ & $X_{n}$ & $X_{p}$ & $X_{\alpha}$ & $X_{\mathrm{Fe}}$ & $X_{\mathrm{Ni}}$ \\
\hline $10^{10}$ & 1.0 & 0.49 & $5.2(-3)$ & $1.8(-2)$ & $5.2(-1)$ & $1.8(-1)$ & $5.0(-4)$ \\
$10^{10}$ & 1.0 & 0.495 & $4.4(-3)$ & $2.1(-2)$ & $5.5(-1)$ & $1.6(-1)$ & $9.5(-4)$ \\
$10^{10}$ & 1.0 & 0.50 & $3.7(-3)$ & $2.5(-2)$ & $5.7(-1)$ & $1.4(-1)$ & $1.6(-3)$ \\
$10^{9}$ & 1.0 & 0.49 & $7.2(-2)$ & $5.2(-2)$ & $8.8(-1)$ & $5.6(-10)$ & $7.3(-14)$ \\
$10^{9}$ & 1.0 & 0.495 & $6.6(-2)$ & $5.6(-2)$ & $8.8(-1)$ & $4.2(-10)$ & $7.4(-14)$ \\
$10^{9}$ & 0.5 & 0.50 & $1.1(-8)$ & $4.2(-3)$ & $5.4(-3)$ & $8.0(-5)$ & $7.7(-1)$ \\
$10^{8}$ & 1.0 & 0.49 & $3.0(-1)$ & $2.8(-1)$ & $4.3(-1)$ & $1.4(-27)$ & $3.0(-31)$ \\
$10^{8}$ & 1.0 & 0.495 & $2.9(-1)$ & $2.8(-1)$ & $4.3(-1)$ & $1.3(-27)$ & $3.1(-31)$ \\
$10^{8}$ & 0.5 & 0.49 & $5.2(-7)$ & $7.3(-3)$ & $4.1(-2)$ & $1.3(-2)$ & $1.6(-1)$ \\
$10^{8}$ & 0.5 & 0.495 & $3.5(-7)$ & $1.1(-2)$ & $4.2(-2)$ & $4.8(-3)$ & $2.8(-1)$ \\
$10^{8}$ & 0.3 & 0.50 & 0.0 & $5.5(-5)$ & $5.3(-6)$ & $8.7(-10)$ & $9.9(-1)$ \\
\hline
\end{tabular}

We need to emphasize the fact that the estimate of neutron density in the relevant region is subject to considerable uncertainty. There is as yet no successful model of supernova explosion. The assumption of NSE is approximate, but should 
not be unreasonable given that silicon burning to $\mathrm{Ni}$ is explosive and takes place at temperatures $T>0.5 \mathrm{MeV}$. In reality we would need to consider all $(n, \gamma)$ reactions that occur in the nucleosynthetic process and we have tried to be conservative in our assumptions in order to derive a meaningful limit. The time available in the neutron bath and the density trajectory of the ejected material is a complicated function of explosion details. We believe however that these effects should not change the result quoted by more than a factor of 100 . It is important to note that, even for the worst case scenario, we can expect a meaningful bound on $\beta^{2}$ for neutrons from these considerations.

Table 1 compares this result with other PV limits. The closest comparison is to the work of Plaga [10] who considered the effect of a possible triplet s-wave (hence PV) proton-proton bound state on the solar p-p cycle, achieving a bound of $10^{-15}$ from the (assumed) approximate validity of the standard solar model.

In addition to the large neutron flux, the supernova has a large proton flux. Indeed, as noted above the proton flux is sufficiently large to overcome the coulomb barrier and convert most of the iron to nickel. This tells us that, for protons, the coefficient of $\beta^{2}$ in Equation (3) must be on the order of one (or larger). As in the neutron case, we are assuming that the extra 1-s proton neither inhibits the decays nor is disturbed by them. For the PV case, because the proton will be much more deeply bound, we might expect it to be considerably larger. Here we just assume it is of order 1 . We thus see immediately that the bound on $\beta^{2}$ is just equal to the bound on the abundance of the anomalous isotope, Co*. One could argue a $10^{3}$ enhancement in view of the fact that the abundance of $\mathrm{Co}^{*}$ will be a fraction of the abundance of Fe while the abundance of normal Co is, at least on Earth, $10^{-3}$ that of Fe. There are no long lived Co isotopes besides the stable ${ }^{59} \mathrm{Co}$. The other isotopes are products of shortlived isotopes and also have very short halflives. 
Moreover the decay energies of Co isotopes are much less than the $20 \mathrm{MeV}$ or more that should be gained by putting the final proton in the $1 s$ state of Cobalt. Thus we expect that any detection of $\mathrm{Z}=27$ nucleus with $A \neq 59$ in a non-irradiated target would indicate $C o^{*}$ and hence Pauli violation.

Finally, we note that existing data can be used to set a limit of interest for one PV model. One of us (RNM) has shown that if Pauli violation is characterized by the commutation relations (see [5]) in Eq. (1), then a matrix representation for the annihilation operators is given by

$$
a=\left(\begin{array}{cccc}
0 & 0 & 0 & . . \\
1 & 0 & 0 & . . \\
0 & \beta_{1} & 0 & . . \\
0 & 0 & \beta_{2} & . . \\
. & . & . & . \\
. & . & . & .
\end{array}\right)
$$

with $\beta_{n}^{2}=1+q+\ldots+q^{n}$. For $q$ close to -1 , one sees that for odd $n, \beta^{n}$ is of the order $\beta_{1}^{(n+1) / 2}$ while, for even $n, \beta_{n}$ is of order 1 . Thus, in a neutron rich environment, the second neutron is added relatively easily. The presupernova star has an oxygen shell with a neutron abundance around $n \sim 10^{20}$. Eq (3) gives a capture probability of about $0.1 \beta^{2}$. Middleton et al, some time ago, put a mass spectrometer limit on on anomalous oxygen, down to 20 AMU of about $O^{*} / O<$ $10^{-18}$ which gives a PV bound, within the model of [5], of $\beta_{1}^{2}<10^{-17}$. We note however that the above matrix representation was derived in [5] for the case of a single oscillator. It is therefore not clear whether it will remain valid in an actual field theory generalization. One possible argument which may suggest its validity in a general field theory, can be given as follows. If we put the quon fields in a thermal bath, in the low temperature limit, we expect the partition function for the system to be dominated by the lowest energy level which will then be described by a single 
oscillator. For this case then the single oscillator representation should remain valid. In summary, we suggest use of mass spectrometry to limit Pauli principle violations for neutrons and protons by exploiting the high nucleon fluxes in supernovae. A neutron limit of interest on a specific model is derived from existing data on Oxygen isotopes and much lower, model-independent limits from new experiments with iron and cobalt are possible.

\section{ACKNOWLEDGMENTS}

We have benefited greatly from conversations with J. Cowan, D. Elmore, E. Fischbach, and O.W. Greenberg. The work of R. N. M. is supported by the National Science foundation under grant PHY-9802551 and that of V. L. T. by DOE under grant DE-FG03-95ER40908. The work of E. B. is supported in part by NSF grants AST-9417242, AST-9731450; NASA grant NAG 5-3505, and an IBM SUR grant.

\section{References}

[1] M. Goldhaber and G. Scharff-Goldhaber, Phys. Rev. 73, 1472 (1948).

[2] O. W. Greenberg and R. N. Mohapatra, Phys. Rev. D 39, 2032 (1989).

[3] E. Fishbach, T. Kirsten and O. Shaeffer, Phys. Rev. Lett. 20, 1012 (1968).

[4] A. Yu Ignatiev and V. Kuzmin, Sov. Journ. Nucl. Physics, 46, 444 (1987); O. W. Greenberg and R. N. Mohapatra, Phys. Rev. Lett. 59, 2507 (1987); ibid 62, 712 (1989); L. Okun, JETP Lett. 46, 529 (1987); A. V. Gorkov, Phys. Lett. A 137, 7 (1989).

[5] O. W. Greenberg, Phys. Rev. Lett. 64, 705 (1990); R. N. Mohapatra, Phys. Lett. 242, 407 (1990); D. I. Fivel, Phys. Rev. Lett. 65, 3361 (1990). 
[6] O. W. Greenberg, Phys. Rev. D 43, 4111 (1991); Physica A 180, 419 (1992);

O. W. Greenberg and R. Hilborn, hep-th/9808106.

[7] K. Deilamian, J. D. Gillaspy and D. Kelleher, Phys. Rev. Lett. 74, 4787 (1995);

K. Deilamian, Ph. D. thesis, Univ. of Wisconsin, Madison, 1991.

[8] E. Ramberg and G. Snow, Phys. Lett. B 238, 438 (1990).

[9] E. Nolte et al., Technical Univ. of Munich preprint (1989).

[10] R. Plaga, Z. Phys. A 333, 397 (1990).

[11] A. Ignatiev, G. C. Joshi and M. Matsuda, UM-P-94/62;

[12] R. Hilborn and Candice L. Yuca, Phys. Rev. Lett. 76, 2844 (1996); M. d Angelis, G. Gagliardi and L. Gianfrani, Phys. Rev. Lett. 76, 2840 (1996).

[13] T. K. Hemmick et al., Phys. Rev. D 41, 2074 (1990). R. Middleton et al. Phys. Rev. Lett. 43, 429 (1979); R. Middleton et al. ANL-PHY-81-1 (1981).

[14] F.-K. Thielemann and K. Nomoto and M. Hashimoto, 1998, ApJ, 460, 408; F. Timmes and S. Woosley and T. Weaver, 1995, ApJS, 98, 617; T. Tsujimoto and K. Nomoto and Y. Yoshi and M. Hashimoto and S. Yanagida and F.-K. Thilemann, 1995, MNRAS, 277, 945.

[15] J. Blatt and V. Weisskopf, Theoretical Nuclear Physics, Dover (1988).

[16] J. Cooperstein and E. Baron in Supernovae, A. Petschek, ed., Springer-Verlag, New York, 1990, p. 213; J. Cooperstein, Nucl. Phys. A 438, 722 (1985); E. Baron and J. Cooperstein and S. Kahana, Nucl. Phys. A 440, 744 (1985); Phys. Rev. Lett. 55, 126 (1985). 
[17] S. Woosley and R. Hoffman, ApJ 395, 202 (1992); B. Meyer, T. Krishnan, and D. Clayton, ApJ 498, 808 (1998). 